\title{
DESCRIPCIÓN DE LA PERCEPCIÓN DEL HABLA EMOCIONAL INFANTIL: TEORÍA Y PRÁCTICA
}

\author{
OLGA ABAKUMOVA Y HERMENEGILDO LÓPEZ GONZÁLEZ
}

Universidad Estatal de Voronezh (Rusia) y Universidad de León

Bajo el concepto de "percepción" el Diccionario de la RAE señala la "sensación interior que resulta de una impresión material hecha en nuestros sentidos". Para Golovin sería, de forma más concreta, una "reflexión entera de objetos, fenómenos, situaciones y eventos en correlación sensible de tiempo y espacio" (Golovin, 1988: 86).

Entendido tal como nos plantea este último aserto, el tema de la percepción del habla presenta un gran interés para ciencias tales como la fisiología, la neurología, la psicología y la psicolingüística.

\section{La percepción del habla como proceso fisiológico.}

Todos los mecanismos que forman parte del proceso de formación del habla se dividen, según N. I. Zhinkin, en dos grupos: mecanismos de percepción del habla y mecanismos de producción del habla, que a su vez se componen de una serie de sistemas. La percepción del habla empieza por el oído, donde los impulsos sonoros actúan sobre los puntos sensibles que se sitúan a lo largo de la membrana basilar de la cóclea, responsable, pues, de la respuesta en frecuencia del oído humano. Cada punto sensible de esa membrana se relaciona con ciertos filamentos nerviosos que pasan de inmediato al cerebro a través del nervio auditivo. Con todo esto la percepción del habla se considera como una esfera especial en la cual se aprecia no el objeto, sino su símbolo. El proceso de percepción se realiza a través de una especie de tamiz y está relacionado con el analizador auditivo.

A la hora de investigar la percepción se deben analizar los siguientes aspectos: 1) formación de los órganos del sentido; 2) atención; 3) órganos del sentido (sentido del gusto, del olfato, del oído y de la vista); 4) procesos de percepción en todos los sentidos; 5) ilusión y constancia de la percepción.

N. I. Zhinkin considera que

la presencia del análisis y síntesis doble señala al habla, de forma destacada, entre todos los demás tipos de la actividad humana. La percepción visual también se caracteriza por el análisis y síntesis inicial del espacio, color y luz en el receptor óptico. La síntesis continúa en la parte cortical; sin embargo el segundo análisis y síntesis no tienen lugar (Zhinkin, 1958: 131).

La percepción del habla se realiza mediante los analizadores auditivo y sonoro. El proceso analítico-sintético consta de dos fases: en la primera la palabra se transmite al analizador auditivo donde se descompone en elementos sonoros y vuelve a formar una integridad compleja; esta fase presupone la 
percepción de la palabra. La segunda fase, de mayor complejidad, consiste en que el estímulo, formado en el analizador auditivo, se extiende discrecionalmente. Dicha fase presupone la comprensión de la palabra y del habla en su totalidad.

Cada uno de nuestros órganos del sentido es parecido a un tamiz que retiene solo una mínima parte de toda la información accesible. Nuestra visión del mundo está, entonces, limitada, dada la limitación del diapasón de las fuerzas a las que somos capaces de reaccionar. Somos capaces de percibir solo las fuerzas que nuestros órganos del sentido pueden detectar y convertir en impulsos nerviosos (Bekeshi, 1974: 58).

Por ello, podemos afirmar que la selección de la información viene limitada por la organización de los correspondientes órganos del sentido.

A la hora de investigar el habla se distinguen los siguientes tipos de percepción: percepción auditiva y percepción visual. El presente artículo, en cuanto que nos interesamos especialmente por la transmisión de la lengua vía oral, está enfocado más a la percepción auditiva.

Es importante, como punto de partida, tomar en consideración dos canales de percepción a través de los órganos del oído y de la conductividad ósea. Si la persona oye sonidos, solo conductibles por el aire, los componentes de baja frecuencia de vibración de las cuerdas vocales se pierden. Esto explica el hecho de que la persona experimenta dificultades en reconocer su propia voz en una grabación. Así,

a la hora de percibir nuestra propia voz, las vibraciones de baja frecuencia de nuestras cuerdas vocales, que se transmiten a la cóclea a través del hueso, proporcionan más fuerza y dinamismo a los sonidos de nuestra propia habla, en comparación con otras ondas sonoras que se transmiten al oyente o se producen en una grabación (Bekeshi, 1974: 113).

D. Bekeshi, por otro lado, y en su artículo Mecanismos y módulos, argumenta sobre la relación inversa de la voz. El control del habla y el canto es impensable sin la participación del sistema complejo de la relación inversa. El sistema de la relación inversa entre los ojos y los músculos controla los movimientos de la mano, por ejemplo, cuando el ser humano se dispone a coger algún objeto, y regula constantemente los parámetros de la voz durante el habla o el canto. El principio del sonido comienza desde el punto de vista de su altura, y si el sonido producido es incorrecto, la tensión de las cuerdas vocales cambia inmediatamente. Dicha relación inversa requiere un mecanismo extremadamente complejo y rápido. El funcionamiento de este mecanismo aún no está estudiado por completo; no obstante, no es de sorprender, a la luz de estas afirmaciones, el hecho de que un niño tarde tan solo unos años en aprender a hablar, mientras que para un adulto aprender a hablar una lengua sin acento es prácticamente imposible. Cualquier contravención en la relación inversa conlleva trastornos del habla.

Las particularidades de la percepción visual están descritas por U. Neiser en su conocido artículo Procesos visuales, donde el autor presta mucha atención al carácter selectivo del uso de la información recibida por el ojo. Aunque los ojos 
se llaman, comúnmente, los espejos del alma, no solo son "mirillas", sino también una especie de entrada, a través de la cual penetra toda una serie de material todavía no tratado para el sistema constructivo visual.

La idea de la correlación de la actividad de los analizadores auditivo y visual permite entender los mecanismos de percepción del habla. Dichos mecanismos se forman en un niño a partir de una relación compleja de muchos analizadores, como resultado de la influencia del habla de los adultos. El estímulo verbal es real, como todos los demás, pero más global. N. I. Zhinkin, en el análisis del estímulo verbal, habla de la hora del accionamiento de cada uno de los analizadores. La comunicación entre los seres humanos mediante el habla solo es posible si los funcionamientos del analizador auditivo y el analizador motriz están completamente coordinados. Es importante añadir, sin embargo, que en el habla infantil la percepción visual tiene un papel fundamental.

En la psicología clásica bajo el concepto de "percepción" se entiende el proceso de análisis del objeto disponible. Al desaparecer el objeto, se activa la memoria, que guarda la imagen de esto objeto. Teniendo como base esta afirmación primera, tomada del trabajo de N. I. Zhinkin, El habla como conducto de información, intentaremos, a través de esta reflexión y con el apoyo de nuestra investigación práctica, encontrar algunas respuestas al problema planteado.

Sabido es que existe una memoria a largo plazo y una memoria a corto plazo, denominada también memoria operativa. Para entender lo que se ha dicho es necesario realizar el análisis sintáctico de la frase de la manera que lo hace un lingüista. En realidad es imposible entender el habla si el objeto de la percepción está ausente o si el oyente no se ha encontrado nunca con él, como pasa a menudo con la percepción del habla extranjera. No obstante, la realización de dicho análisis es imposible, no solo por la alta velocidad del flujo de las palabras, sino también por la gran cantidad de formas verbales y reglas de su combinación que se deben interiorizar. Esto nos lleva a la conclusión de que la memoria verbal sería la que hemos denominado a largo plazo. La memoria a corto plazo, de 10 segundos, no es suficiente para realizar este análisis. Los viejos errores de los investigadores de la percepción del habla consistían en el hecho de partir de una idea errónea: que los componentes del sistema de signos se pueden reconocer durante el mismo proceso de percepción, sin recurrir a la memoria a largo plazo, que constituye la base de la experiencia de reconocimiento y del mecanismo automático de presentación del material reconocido.

La percepción y sus propiedades dependen, por un lado, del objeto y, por el otro, de la memoria a largo plazo, ya que el mecanismo codificador y el mecanismo decodificador no son capaces de crear el código a causa de la complejidad del objeto real. Los códigos no son mecanismos hechos, sino que precisan ser creados en la comunicación. Es importante aprender a mirar, ver, oír y sentir.

Por tanto, la percepción del habla por un ser humano no es un proceso simple. La facilidad con la cual la persona reconoce palabras y entiende frases es la última fase del complejo proceso de procesamiento del sistema de signos. 
La percepción de una lengua extranjera presenta interés y no solo desde el punto de vista de la metodología de la enseñanza, puesto que la comprensión de dichos procesos permite profundizar en su esencia, cuando se trata del paso a otro sistema de signos y de la interacción de dos sistemas: materno y no materno. I. N. Gorelov sostiene que

la complejidad del proceso de percepción del sentido está condicionada por el hecho de que cada palabra, en el habla, presenta propiedades tales como la polisemia y la homonimia. El modelo lineal simplificado de la comprensión del habla se puede observar solo en caso de la percepción de un texto en lengua extranjera. Los que aprenden una lengua extranjera tienen, con excesiva frecuencia, la dificultad de identificar el significado de los lexemas que parecen no formar frases completas. Esta dificultad es superable si el aprendizaje de una lengua parte del método comunicativo que hace uso de varios contextos verbales y situaciones en los que se utilizan los elementos de una lengua. Los contextos verbales y situaciones comunicativas concretas, en el curso de la percepción del habla materna, permiten al destinatario del mensaje escoger, de una multitud de significados de la palabra, el que sobreentiende el hablante (Gorelov, 1997: 86).

La importancia dada, por determinadas metodologías, al aspecto léxicogramatical de la decodificación, nos lleva a concluir que, simplemente conocer un idioma, desde esa perspectiva, es insuficiente para la percepción completa del mensaje verbal. Es muy importante la referencia adecuada, es decir, la relación con la situación real. I. N. Gorelov subraya que N. I. Zhinkin tiene razón cuando afirma que "entendemos no el habla (el texto), sino la realidad" (Gorelov, 1997: 87).

Un considerable papel en el proceso de la percepción del mensaje lo tiene la actividad mental del destinatario del mensaje que éste encuentra y que activa el mecanismo de pronosticación de la comprensión del habla.

Todo lo anteriormente dicho hace referencia al habla materna. La percepción del habla extranjera debe ser un proceso de mayor complejidad en el que el oyente tiene que ver no solo con el sistema de otra lengua, sino también con el sistema de la lengua materna, que entran en interacción. El primero en prestar atención a la subjetividad de la percepción del habla extranjera fue E. D. Polivanov. El citado científico presenta la famosa tesis sobre el carácter subjetivo de la percepción del habla extranjera:

Los fonemas y otros elementos fonológicos elementales están tan fuertemente asociados por cada hablante de su lengua materna a su actividad perceptiva (es decir, a los actos de la percepción del habla), que dicha persona se inclina a analizar el habla según su sistema de elementos fonológicos - incluso en el caso de una lengua extranjera. Dicho con otras palabras, al oír una palabra extranjera desconocida (o una frase del habla extranjera que podría ser captada por la atención auditiva), el oyente intenta encontrar en ella el sistema de sus elementos fonológicos y descomponer el habla en sus fonemas y en las reglas de su combinación según el sistema de su lengua materna (Polivanov, 1968: 236).

Como consecuencia de ello, la percepción fonológica de hablantes de distintas lenguas tiene un carácter subjetivo, subrayando que esta subjetividad y estas diferencias en la percepción del mismo grupo de sonidos por varias 
mentalidades no dependen de las particularidades de cierta raza humana, sino de un sistema de hábitos de lengua, formados por cada individuo durante el aprendizaje de su lengua materna (Polivanov, 1968: 246).

Para el presente trabajo, el mayor interés viene representado por el problema de la percepción infantil, o sea, su formación y el carácter emocional tanto de la producción como de la percepción del habla. L. S. Vigotsky en su artículo Percepción y desarrollo en la edad infantil (1997) subraya que, en el proceso del desarrollo infantil, la función de la percepción se relaciona con la memoria eidética y, mediante esto, aparece un todo nuevo único, en el cual la percepción funciona como una parte interior; confluyen así las funciones del pensamiento visual y las de la percepción, a tal grado que ya no somos capaces de diferenciar la percepción categorial de la percepción inmediata, es decir, de la percepción del objeto como tal, separadamente de su significado. La experiencia indica que, en este punto, aparece la relación del habla y la palabra con la percepción, donde cambia el proceso de la percepción habitual para el niño, mirado dicho proceso a través del prisma del habla, si el niño no solo percibe el mensaje, sino también lo produce. Estas relaciones multifuncionales se observan a menudo, y, gracias a la aparición de nuevas relaciones entre la percepción y las demás funciones, son posibles cambios importantes y propiedades de la percepción desarrollada de adultos que son inexplicables, si la evolución de la percepción se analiza aisladamente y no como una parte del desarrollo de la conciencia en general.

Investigaciones, suficientemente probadas y asentadas en la comunidad científica, demuestran que el carácter de las relaciones multifuncionales consiste en lo siguiente: en las primeras etapas del desarrollo, la percepción está ligada a la actividad motórica formando solo una parte de todo el proceso sensomotórico. Con el paso del tiempo, y muy poco a poco, la percepción va ganando independencia separándose de esta ligazón con la actividad motórica. Según el psicólogo alemán K. Levin, que dedicó una considerable parte de su tiempo a los estudios relacionados con este aspecto concreto, la percepción de un niño se expresa dinámicamente en una serie de procesos interiores solo al pasar unos años. En particular, dicho estudioso afirma que la relación entre el pensamiento visual y la percepción es posible solo después de que ésta haya cesado de formar parte de todo este proceso sensomotórico.

T. P. Hrizman, V. P. Eremeeva y T. D. Loskutova en su trabajo Emociones, habla y actividad del cerebro infantil (1991) realizaron unos experimentos para estudiar el proceso de percepción en niños de 3 a 7 años que desarrollaban el proceso de percepción y comprensión, por ejemplo y para el caso que nos ocupa, de señales verbales fuera del texto $y$, en concreto, en el contexto de una historia o fábula. Cuando los datos del experimento fueron recopilados, se llegó a la conclusión de que el cerebro de un niño pequeño se activa más cuando la palabra tiene para el niño un significado personal, es decir, cuando la misma adquiere un carácter emocionalmente significativo. Es de notar que la reacción activa y selectiva del niño hacia estímulos verbales se forma como resultado de la inclusión y perfección de los mecanismos de formación del significado a nivel de los sistemas corticales. 
Por lo tanto existe una dependencia funcional entre la direccionalidad y el carácter selectivo de los procesos psíquicos y los mecanismos de comprensión del habla. El papel decisivo en la regulación de éstos pertenece a las áreas frontales de la corteza cerebral, es decir, áreas asociativas, según Leontiev (1969).

Se ha descubierto que, cuando los niños de 3 a 7 años perciben $y$ comprenden señales verbales que tienen un grado diferente del valor emocional, en sus sistemas corticales se produce un cambio importante, relativo a la organización funcional de la interacción intercentral: aumenta el nivel de las relaciones intercentrales de todas las zonas estudiadas, lo que indica que la actividad funcional del cerebro del niño aumenta a la hora de hablar. Aparte de esto, selectivamente, aumenta la interacción dentro y entre los hemisferios cerebrales de las zonas frontales asociativas, de manera especial con las zonas motóricas y temporales. La actividad interrelacionada se enfoca en estas zonas de la corteza cerebral y, en los hemisferios derecho e izquierdo, aumenta la interacción funcional de los centros simétricos de las zonas frontales.

Se ha conseguido probar, asimismo, que los procesos corticales de percepción, análisis semántico y comprensión de una palabra, por un niño, tienen una localización y lateralización dinámica muy compleja, y la inclusión de los componentes emotivos activa dichos procesos y les proporciona un dinamismo especial, tanto en la interacción "hemisferio derecho - izquierdo", como en la de las partes delanteras y traseras de la corteza. Esto se puede constatar en:

a) el aumento de la asimetría funcional (total y parcial) entre los hemisferios y en el cambio de su señal;

b) el aumento selectivo de las relaciones de las partes simétricas de la corteza entre los hemisferios y su redistribución dinámica y;

c) el aumento de la interacción cortical en las partes delanteras de la corteza y la disminución de dicha interacción en las partes traseras.

En el proceso de percepción del habla las emociones activan de manera distinta el hemisferio derecho y el hemisferio izquierdo del niño aumentando y estabilizando la interacción intracortical y sobre todo activando la dinámica de la reorganización de la interacción introcortical en el hemisferio izquierdo.

"En las primeras etapas la percepción es inseparable tanto del proceso sensomotórico como de la reacción emotiva" - según los datos de G. Folkelt, F. Kriuger y otros científicos de Leipzig. Con esta argumentación F. Kriuger, un psicólogo y filósofo alemán, fundador de la escuela de la "psicología íntegra", propuso denominar la percepción en las primeras etapas como "percepción sensible, emotiva". En sus trabajos, demostró que la percepción se va separando del efecto inmediato, de la emoción inmediata del niño solo con el paso del tiempo (Vigotsky, 1997: 27).

A pesar de cuanto venimos afirmando, la percepción del habla en niños de 3 a 7 años es un fenómeno complejo y aún muy poco estudiado. La percepción se realiza de manera diferente en el contexto natural o escolar de la comunicación verbal.

Un gran interés, a nuestro entender, vendría representado por el problema 
de la percepción de la emotividad en niños de edad temprana en su habla materna. Así, y para una primera aproximación al estudio de este fenómeno elegimos, como material de trabajo, un cuento.

Para estudiar la reacción de los niños hacia el habla emotiva y como estímulos emociogénicos utilizamos fragmentos de cuentos mundialmente conocidos, tales como: El Patito Feo de C. Andersen, El Lobo y los siete Cabritillos (cuento popular) y $\mathrm{La}$ Cenicienta de Charles Perrault. Dichos cuentos se caracterizan por un especial contenido social y emotivo y son capaces de causar, a través de la palabra y aún en niños de edad temprana, unas emociones específicamente humanas tales como la alegría, el interés, la pena, el sufrimiento, el miedo o el estado neutro. Como una clasificación de los estados emocionales decidimos utilizar la clasificación de K. Izard, puesto que el autor menciona los tres criterios que caracterizan el estado emocional: biológico, fisiológico y psicológico.

Los autores del presente trabajo se atienen el criterio común de que todas las emociones se dividen en positivas y negativas y se pueden analizar según los vectores correspondientes. Dicho planteamiento está perfectamente ilustrado en algunos trabajos de E. N. Vinarskaya (1989), que sostiene que "la base de las emociones de una persona está constituida por tres modalidades principales: 1 . alegría, 2. pena, 3. ira/miedo", aunque aceptamos como buenas y reconocemos, por lo mismo, algunas otras clasificaciones mucho más amplias, como la de Izard (1977), cuya teoría se conoce bajo la denominación de Teoría diferencial de las emociones, y que el autor basa en trabajos clásicos como el de Darwin, James, etc. Señala, pues, por su parte, Izard 10 emociones básicas: interés, alegría, sorpresa, tristeza, ira, disgusto, desprecio, miedo, vergüenza-timidez y culpabilidad, cada una de ellas definida por tres niveles, a saber: neurofisiológico, expresivo y experiencial. Por operatividad, sin embargo, nos mantendremos en nuestra clasificación primera, la de E. N. Vinarskaya.

A pesar de cuanto venimos afirmando, nuestra investigación no pretendía quedarse en el marco de la pura teoría, y así se anunciaba ya en el propio título de la misma; estamos convencidos de que a la hora de reflexionar sobre el habla emocional infantil es necesario acompañar dicha reflexión de una base sólida, apoyada en la investigación experimental. Así pues, teniendo siempre presentes las ideas arriba expresadas y tratando de encontrar mejores caminos para afrontar con éxito la enseñanza de la lengua (materna o extranjera), nos propusimos llevar a cabo la experiencia que sigue y que, además del preceptivo análisis de los resultados, constaba de dos fases:

1. Cada niño tenía que escuchar un fragmento de uno de los cuentos. Mientras el niño escuchaba se observaban cambios en su comportamiento (su mímica, gestos, acciones, palabras sueltas). A través de los elementos de enseñanza se prestaba atención a la reacción del mismo.

2. En la segunda parte del experimento el niño tenía a su disposición una imagen del fragmento que había escuchado y se estudiaba su reacción emotiva hacia la imagen. 
Al conocer el contenido del cuento y al escuchar algunos fragmentos de éste los niños expresaban unos sentimientos determinados que reflejaban su reacción hacia el texto que estaban escuchando. Para facilitar, de todos modos, la comprensión recurrimos al canal audiovisual; es decir, los niños podían escuchar y comprender los fragmentos de los cuentos al proporcionarles imágenes de los mismos. Se observaba el aspecto expresivo de la reacción de los niños hacia los fragmentos de los cuentos, en los que a los personajes les ocurría toda una serie de aventuras-acontecimientos capaces de causar emociones positivas o negativas. Los niños debían, asimismo, evaluar los sentimientos de los personajes, ya fueran positivos (alegría) o negativos (pena/sufrimiento, miedo). Con todo esto, dependiendo del estado emocional que se atribuía al personaje, los niños indicaban los modelos de: 1. imagen de una persona portadora de emociones positivas (alegría) o negativas (pena/sufrimiento, miedo); 2. color - asociación.

Para el estudio concreto del habla infantil fueron utilizados los siguientes métodos:

- observación de los niños (en particular, del aspecto expresivo de su comportamiento) durante el juego y el proceso de percepción del habla en forma de cuento;

- conversaciones con los niños, durante las cuales se dialogaba sobre su actitud hacia los fragmentos escuchados;

- criterios evaluativos para estudiar las reacciones emocionales, individuales de cada niño.

La adecuación de los sentimientos del niño a las características de la parte expresiva del habla se analizaba a partir de una serie de criterios. En particular, a los niños de 3 a 7 años, en la etapa educativa, se les ofrecían algunas maneras de expresar su actitud hacia el cuento. Para los niños de 6 o 7 años fueron escogidos unos indicadores de color específicos. Los niños aprendían la manera de expresar su estado emocional mediante un color determinado:

\begin{tabular}{|c|c|c|}
\hline Emoción & Estado emocional & Color \\
\hline alegría & estoy alegre & rojo \\
\hline pena/sufrimiento & me da pena & marrón \\
\hline- & neutro & blanco \\
\hline interés & me interesa & rosa \\
\hline miedo & me da miedo & negro \\
\hline
\end{tabular}

Los autores del presente trabajo partieron de las nociones tradicionales en la cultura europea sobre la simbología del color.

El color puede tener un significado comunicativo, un significado determinante que establece la relación entre los elementos y objetos de la naturaleza; un significado simbólico que indica un fenómeno, objeto o esencia; un significado expresivo (fuerza expresiva) que transmite cierto sentimiento y suscita emociones correspondientes (Kriulina, 1993: 71). 
Por ejemplo, los colores rojo y rosa reflejan emociones positivas tales como la alegría y el entusiasmo, mientras que los colores negro y marrón son indicadores de un estado emocional negativo (miedo, pena/sufrimiento); el color blanco, el color de la luz, pureza y ligereza, expresa un estado emocional neutro (Yanshina, 1996).

Investigaciones previas han desvelado que los niños de 3 a 5 años no se orientan aún bastante bien entre los indicadores de color, por tanto, en calidad de los criterios del estado emocional de los niños fue utilizada una serie de expresiones mímicas. Las emociones positivas se presentaban en forma de una cara sonriente $: \dot{*}$, las emociones negativas estaban reflejadas por una cara que llora $: \dot{\theta}$, y la ausencia de emociones se expresaba por medio de una cara sin ningún estado emocional $:$, siguiendo, en este caso, los consejos de Temml, Dorki y Amen (1995: 110-121).

Así fueron observadas las reacciones de niños de 3 a 7 años hacia varios fragmentos de los 3 cuentos arriba citados: El Patito Feo, El Lobo y los siete cabritillos y La Cenicienta. La versión auditiva contaba de 253 fragmentos que expresaban sentimientos tipo "alegría" y 404 fragmentos que incluían situaciones capaces de provocar emociones tipo "pena/sufrimiento, miedo". Al incluir el canal visual en el proceso de percepción, hubo 241 fragmentos de emociones positivas (alegría) y 500 fragmentos de emociones negativas (pena/sufrimiento, miedo).

La siguiente tabla determina, de una forma concreta, la dinámica de la percepción adecuada en estos niños, sometidos al experimento, en lo que se refiere a las emociones "alegría", "pena/sufrimiento, miedo", y contemplando el parámetro de la edad.

\begin{tabular}{|c|c|c|c|c|}
\hline$\frac{\bar{T}}{\pi}$ & \multicolumn{4}{|c|}{$\begin{array}{c}\text { Número de casos (в \%) de la percepción adecuada en niños respecto a } \\
\text { los sentimientos de los personajes aparecidos en los cuentos de } \\
\text { referencia }\end{array}$} \\
\hline \multicolumn{3}{|c|}{ Emociones «alegría» } & \multicolumn{2}{|c|}{$\begin{array}{c}\text { Emociones «pena/sufrimiento, } \\
\text { miedo» }\end{array}$} \\
\hline & $\begin{array}{l}\text { En forma } \\
\text { auditiva }\end{array}$ & $\begin{array}{c}\text { En forma } \\
\text { audiovisual }\end{array}$ & $\begin{array}{c}\text { En forma } \\
\text { auditiva }\end{array}$ & $\begin{array}{c}\text { En forma } \\
\text { audiovisual }\end{array}$ \\
\hline 3 años & 79,0 & 89,0 & 43,8 & 60,6 \\
\hline 4 años & 92,0 & 89,0 & 58,8 & 60,3 \\
\hline 5 años & 75,0 & 100,0 & 55,8 & 75,6 \\
\hline 6 años & 65,0 & 86,0 & 56,5 & 66,6 \\
\hline 7 años & 60,0 & 80,0 & 70,2 & 81,3 \\
\hline & 74,2 & 88,8 & 57,2 & 68,9 \\
\hline
\end{tabular}

Tal como se aprecia en la tabla, en la mayoría de los casos, las emociones positivas ("alegría"), en los niños, se adecuan a la situación dada con más frecuencia que las emociones negativas. Cuando se presenta el material estimulante en forma auditiva y audiovisual, en niños de 3 a 5 años, las emociones positivas son más distintas que en niños de 6 a 7 años. Entre los 
niños de 5 años se destaca una correlación moderadamente expresa $(0,38)$ de una situación positiva con el carácter de los fragmentos audiovisuales presentados. En niños de mayor edad, dichos cuentos provocan una respuesta emocional menos fuerte, lo que probablemente se debe al hecho de que estos niños ya están familiarizados con los cuentos. El número de los fragmentos situacionales adecuadamente percibidos, en forma auditiva, como portadores de "pena/sufrimiento, miedo" tiende a aumentarse al pasar de 3 años a 4-6 y 7 años; no obstante no han sido encontradas diferencias fiables de estos indicadores en relación a la edad. Son más frecuentes, sin embargo, los casos de la empatía adecuada del estado emocional de miedo, presentado en forma audiovisual, en niños de 5 a 7 años.

El experimento, que no ha hecho sino comenzar, nos ha permitido, por el momento, destacar algunas regularidades en el proceso de percepción del habla emocional en niños, según las diferentes edades, carácter de la emoción, manera de presentación de la información verbal e interacción de los medios expresivos. Todo ello merecerá el objeto de otras reflexiones en las que trataremos de extraer consecuencias aplicadas a la enseñanza, tanto de la lengua materna como de una lengua extranjera, lo que es, en último término, nuestro objetivo primario.

\section{BIBLIOGRAFÍA}

Bekeshi, D. et al. (1974): Percepción. Mecanismos y módulos, Moscú.

Fernández-Abascal, E. y Martín Díaz, M. D. (1995): “Emociones negativas II: Ira, hostilidad y tristeza", en E. G. Fernández Abascal (Ed.): Manual de Motivación y Emoción, Madrid, Ramón Areces.

Golovin, S. (1998): Diccionario del psicólogo práctico, Minsk, Harvest.

Gorelov, I. N., Sedov K.F. (1997): Principios de psicolingüística, Moscú, Labirint.

Hrizman, T. P., Eremeevaa V.P., Loskutova T.D. (1991): Emociones, habla y actividad del cerebro infantil, Moscú, Pedagogía.

Izard, C. E. (1977): Human emotions, New York, Plenun Press.

Kriulina, A. A. (1993): Color en nuestra vida, Kursk, Kurskinformpechat.

Lazarus, R. S. (1991): Emotion and adaptation, New York, Oxford University Press.

LeDoux, J. (1999): El cerebro emocional, Barcelona, Editorial Planeta.

Leontiev, A.A. (1969): Lenguaje, habla y actividad del habla, Moscú, Prosvezshenie.

Vigotsky, L. S. (1997): Ciclo de conferencias de psicología, Moscú, Unión.

Vinarskaya, E. N. (1989): Medios expresivos del texto, Moscú, Viszhaya shkola.

Yanshina, P. V. (1996): Color emocional, Samara, SamGPU.

Zhinkin, N. I. (1958): Mecanismos del habla, Moscú, APN RSFSR.

Zhinkin, N. I. (1982): El habla como conducto de información, Moscú, Nauka. 